\title{
The mechanism underlying acetaminophen- induced hepatotoxicity in humans and mice involves mitochondrial damage and nuclear DNA fragmentation
}

\author{
Mitchell R. McGill, ${ }^{1}$ Matthew R. Sharpe, ${ }^{2}$ C. David Williams, ${ }^{1}$ Mohammad Taha, ${ }^{2}$ \\ Steven C. Curry, ${ }^{3}$ and Hartmut Jaeschke ${ }^{1}$
}

\begin{abstract}
1Department of Pharmacology, Toxicology, and Therapeutics, University of Kansas Medical Center, Kansas City, Kansas, USA. ${ }^{2}$ Department of Internal Medicine, University of Kansas Hospital, Kansas City, Kansas, USA. ${ }^{3}$ Department of Medical Toxicology, Banner Good Samaritan Medical Center, Department of Medicine, University of Arizona College of Medicine, Phoenix, Arizona, USA.
\end{abstract}

\begin{abstract}
Acetaminophen (APAP) overdose is the predominant cause of acute liver failure in the United States. Toxicity begins with a reactive metabolite that binds to proteins. In rodents, this leads to mitochondrial dysfunction and nuclear DNA fragmentation, resulting in necrotic cell death. While APAP metabolism is similar in humans, the later events resulting in toxicity have not been investigated in patients. In this study, levels of biomarkers of mitochondrial damage (glutamate dehydrogenase [GDH] and mitochondrial DNA [mtDNA]) and nuclear DNA fragments were measured in plasma from APAP-overdose patients. Overdose patients with no or minimal hepatic injury who had normal liver function tests (LTs) (referred to herein as the normal LT group) and healthy volunteers served as controls. Peak GDH activity and mtDNA concentration were increased in plasma from patients with abnormal LT. Peak nuclear DNA fragmentation in the abnormal LT cohort was also increased over that of controls. Parallel studies in mice revealed that these plasma biomarkers correlated well with tissue injury. Caspase-3 activity and cleaved caspase- 3 were not detectable in plasma from overdose patients or mice, but were elevated after TNF-induced apoptosis, indicating that APAP overdose does not cause apoptosis. Thus, our results suggest that mitochondrial damage and nuclear DNA fragmentation are likely to be critical events in APAP hepatotoxicity in humans, resulting in necrotic cell death.
\end{abstract}

\section{Introduction}

Acetaminophen (APAP) is one of the best-selling analgesics in the US (1-3) and one of the most popular drugs in the world. Although it is safe at therapeutic doses, overdose of APAP can cause severe liver injury. The first reports of APAP hepatotoxicity in humans appeared in the literature in the 1960s (4). Since that time, APAP overdose has become the most common cause of acute liver failure in most Western countries (5-8). In the US alone, misuse of APAP is responsible for about 56,000 emergency room visits, 26,000 hospitalizations, and nearly 500 deaths each year $(5,9)$. Approximately half of these cases are the result of intentional overdosing, with higher proportions in other countries. These patients typically present to the emergency department at the start or near the peak of injury, with rapidly increasing liver enzymes in plasma.

Over the last 4 decades, considerable progress has been made in rodent models toward understanding the mechanisms of APAP hepatotoxicity. The majority of a therapeutic dose (>90\%) of APAP is glucuronidated or sulfated and then excreted. A small percentage is metabolized by cytochrome P450 enzymes (CYP) to the reactive intermediate $\mathrm{N}$-acetyl-p-benzoquinone imine (NAPQI), which is readily detoxified by conjugation with glutathione (GSH) (10). From rodent studies, we know that much higher doses saturate the glucuronidation and sulfation pathways, resulting in formation

Conflict of interest: Steven C. Curry and Hartmut Jaeschke are supported by grants from McNeil Consumer Health.

Citation for this article: J Clin Invest. 2012;122(4):1574-1583. doi:10.1172/JCI59755 of excess NAPQI. The additional reactive metabolite depletes liver GSH and binds to proteins (11-14). Ultrastructural and biochemical studies demonstrated that toxic doses of APAP could cause changes in the morphology and function of liver mitochondria (15, 16). While it was clear from these data that mitochondrial injury occurs after treatment with large doses of APAP, it was not known how this developed. Important insight came from 2 sources. First, a comparison of APAP and the nonhepatotoxic regioisomer 3 '-hydroxyacetanilide, which can also bind to proteins, revealed that APAP binds more frequently to mitochondrial proteins (17). Second, it was found that APAP treatment could cause mitochondrial oxidative stress in mice (18). This suggested that NAPQI binding to mitochondrial proteins leads to mitochondrial oxidative stress. It is now known that this causes the mitochondrial membrane permeability transition (MPT) pore opening (19-21), matrix swelling, and outer membrane lysis in rodent models (15). The permeabilization and lysis result in the release of apoptosis-inducing factor (AIF) and endonuclease $\mathrm{G}$ (EndoG) from mitochondria. These endonucleases translocate to nuclei and cause nuclear DNA fragmentation (22). Proapoptotic proteins, including cytochrome $c$ and Smac/DIABLO, are also released. However, there is no activation of caspases, and caspase inhibitors do not protect against APAP toxicity (23-25). Most likely, ATP depletion prevents activation of the effector caspase- 3 by the apoptosome. The end result is centrilobular hepatocyte necrosis and liver failure $(4,23)$.

In contrast to rodents, understanding of the mechanisms of APAP hepatotoxicity in humans is limited. There is evidence for 


\section{Table 1}

Clinical data on acetaminophen overdose patients

\begin{tabular}{|c|c|c|c|c|c|c|c|c|c|c|c|}
\hline \multirow[b]{2}{*}{ Parameter } & \multicolumn{4}{|c|}{$\begin{array}{l}\text { Abnormal LT group }{ }^{A} \\
\qquad(n=20)\end{array}$} & \multicolumn{4}{|c|}{$\begin{array}{l}\text { Normal LT group } \\
\quad(n=20)\end{array}$} & \multicolumn{3}{|c|}{$\begin{array}{l}\text { Healthy volunteers } \\
\qquad(n=6)\end{array}$} \\
\hline & Range & Mean & Parameter & $\%$ & Range & Mean & Parameter & $\%$ & Range & Mean & $\begin{array}{c}\text { Sex } \\
(\% F)\end{array}$ \\
\hline Age & $19-65$ & 38 & & & $18-56$ & 29 & & & $23-51$ & 36 & \\
\hline Admission APAP $(\mu \mathrm{g} / \mathrm{ml})^{\mathrm{C}}$ & $0-136$ & 40 & $\operatorname{Sex}(F)$ & 65 & $11-582$ & 162 & $\operatorname{Sex}(F)$ & 70 & ND & ND & 66 \\
\hline Peak ALT (U/I)D & 1,137-8,545 & 5,330 & & & 14-158 & 54 & & & $14-31$ & 25 & \\
\hline Peak AST $(\mathrm{U} / \mathrm{I})^{\mathrm{D}}$ & $486-26,000$ & 8,527 & & & $5-300$ & 64 & & & $4-48$ & 27 & \\
\hline Peak PTD & $18.7-374$ & 59.2 & Survived & 95 & $13.1-17.5$ & 15.3 & Survived & 100 & ND & ND & \\
\hline Peak bilirubin $(\mathrm{mg} / \mathrm{dl})^{\mathrm{D}}$ & $0.9-25$ & 7.5 & & & $0.2-1.9$ & 0.9 & & & ND & ND & \\
\hline Peak creatinine $(\mathrm{mg} / \mathrm{dl})^{\mathrm{D}}$ & $0.6-7.8$ & 2.5 & & & $0.4-2.1$ & 0.9 & & & ND & ND & \\
\hline Encephalopathy grade $\geq 1$ & & & & $6 / 20$ & & & & $2 / 20$ & & & \\
\hline
\end{tabular}

APeak $A L T \geq 1,000 \mathrm{U} / \mathrm{l}$ and peak $\mathrm{PT} \geq 18 \mathrm{~s}$. ${ }^{\mathrm{B} P e a k} \mathrm{ALT}<1,000 \mathrm{U} / \mathrm{l}$ and peak $\mathrm{PT}<18 \mathrm{~s}$. CWhen available. ${ }^{\mathrm{P} P e a k}$ value achieved during hospital stay. ND, not determined.

a stress on the hepatic GSH pool after APAP exposure $(26,27)$. In addition, APAP protein adducts are present in plasma after APAP overdose $(28,29)$. These data indicate that metabolic activation and protein adduct formation occur in humans in a manner similar to what has been described in rodents. However, the later events, including mitochondrial dysfunction, DNA fragmentation, and mode of cell death have not been investigated in the clinic. Although studies using the metabolically competent human liver cell line HepaRG provided evidence that the mechanisms of injury in human hepatocytes are similar to those in rodent models (25), cell lines may not reflect in vivo mechanisms.

Currently, the only effective therapy for APAP overdose is GSH replacement in order to scavenge NAPQI, which is accomplished with N-acetyl-L-cysteine (NAC) and other sulfhydryl donors. However, NAC needs to be given within 12 to 24 hours of APAP ingestion. Patients presenting later may benefit from increased metabolic flux (30), but the likelihood of a positive outcome is notably decreased (31). A better understanding of the later events in human APAP toxicity may lead to the development of more effective interventions for late-presenting patients. Therefore the purpose of this study was to gain further insight into the mechanisms of APAPinduced liver injury in humans by investigating whether or not mitochondrial injury and nuclear DNA fragmentation occur as part of the mechanism of toxicity. In addition, we sought direct evidence for caspase activation in overdose patients, as there are somewhat conflicting data regarding the relevance of caspase-dependent apoptosis in the pathophysiology based on the caspase-dependent cleavage of cytokeratin-18 (K18) $(32,33)$. We hypothesized that, if mitochondrial injury and nuclear DNA fragmentation occur, mitochondrial contents and DNA fragments lost into the cytosol as a result of the MPT, mitochondrial lysis, and DNA fragmentation should be detectable in plasma when the cells become necrotic. Furthermore, if relevant apoptotic cell death should occur, active fragments of caspase- 3 and increased caspase- 3 enzyme activity should be detectable in plasma of APAP-overdose patients.

\section{Results}

Study design and patient information. Samples from 40 patients were used in this study: 20 patients with maximum alanine aminotransferase (ALT) of 1,000 U/l or more and maximum prothrombin time (PT) of $18 \mathrm{~s}$ or more (abnormal liver function test [LT] group) and
20 with maximum ALT of less than 1,000 U/l and maximum PT of less than $18 \mathrm{~s}$ (defined as the normal LT group), the latter group having no or minimal hepatic injury. Blood samples were obtained from 6 volunteers as healthy controls. For the abnormal LT cohort, the mean age was 38 years, with a range from 19 to 65 (Table 1). The percentage of male and female patients in the cohorts with normal and abnormal LTs was similar (70\% females vs. $65 \%$ females, respectively). The average peak ALT value for the group with elevated LT values was 5,330 $\pm 562 \mathrm{U} / \mathrm{l}$. Among these 20 patients, 1 did not survive. The remaining patients survived without liver transplant. For the normal LT cohort, the mean age was 29 years, with a range from 18 to 56 . Survival in this group was $100 \%$. The healthy volunteer group was matched to these 2 cohorts, $66 \%$ females with a mean age of 36 and a range of 23 to 51 (Table 1). Interestingly, average serum APAP levels in the normal LT group were markedly higher than in the abnormal LT group. This is likely because many of the patients in this group presented earlier, before extensive APAP metabolism. Earlier admission and treatment would explain why these patients did not develop signs of liver injury.

Glutamate dehydrogenase activity in plasma from overdose patients. We reasoned that, if mitochondrial injury occurs in humans as it does in rodents, mitochondrial contents released into the cytosol after the MPT and mitochondrial membrane lysis should become detectable in the plasma of the injured patients after cells become necrotic. With this in mind, we measured the activity of the mitochondrial enzyme glutamate dehydrogenase (GDH) in the plasma obtained from each cohort. The GDHs are enzymes of the mitochondrial matrix that are highly expressed in the liver (34). These enzymes catalyze the conversion of 2-oxoglutarate to L-glutamate using $\mathrm{NADH}$ and $\mathrm{NH}_{4}{ }^{+}$. We observed clear increases in GDH activity over time in patients with abnormal LTs (Figure 1, A-C). The average GDH activities were $22 \pm 7,10 \pm 3$, and $552 \pm 113 \mathrm{U} / \mathrm{l}$ for healthy volunteers, the normal LT group, and the abnormal LT group, respectively (Figure 1D). Additionally, the average increase in GDH activity in patients with abnormal LTs correlated with the rise in ALT values (Pearson's coefficient $=0.45 ; P<0.05$ ) (Figure 1, $\mathrm{E}$ and $\mathrm{F})$. Although the average PT values (59.2 s) in patients with liver injury (high ALT) were almost 4 times higher than in patients with no relevant ALT increase (15.3 s), there was no significant correlation between peak GDH activity and PT values in the abnormal LT group (data not shown). 
A
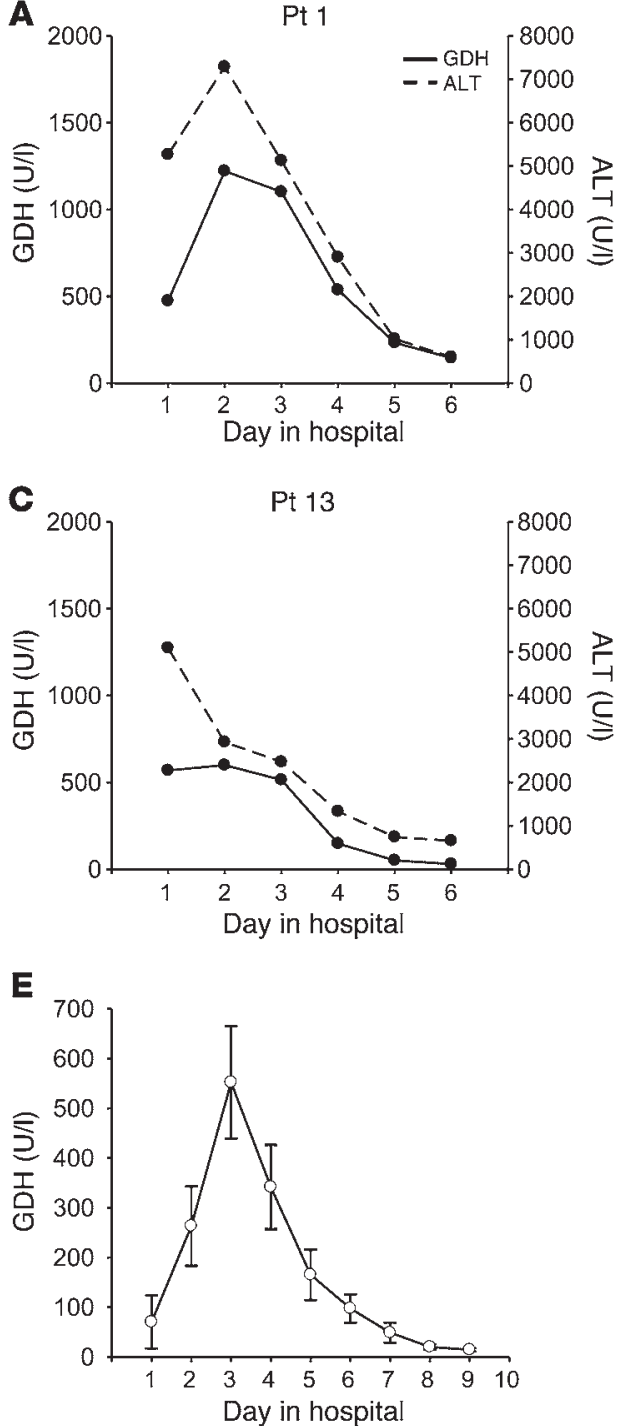

B
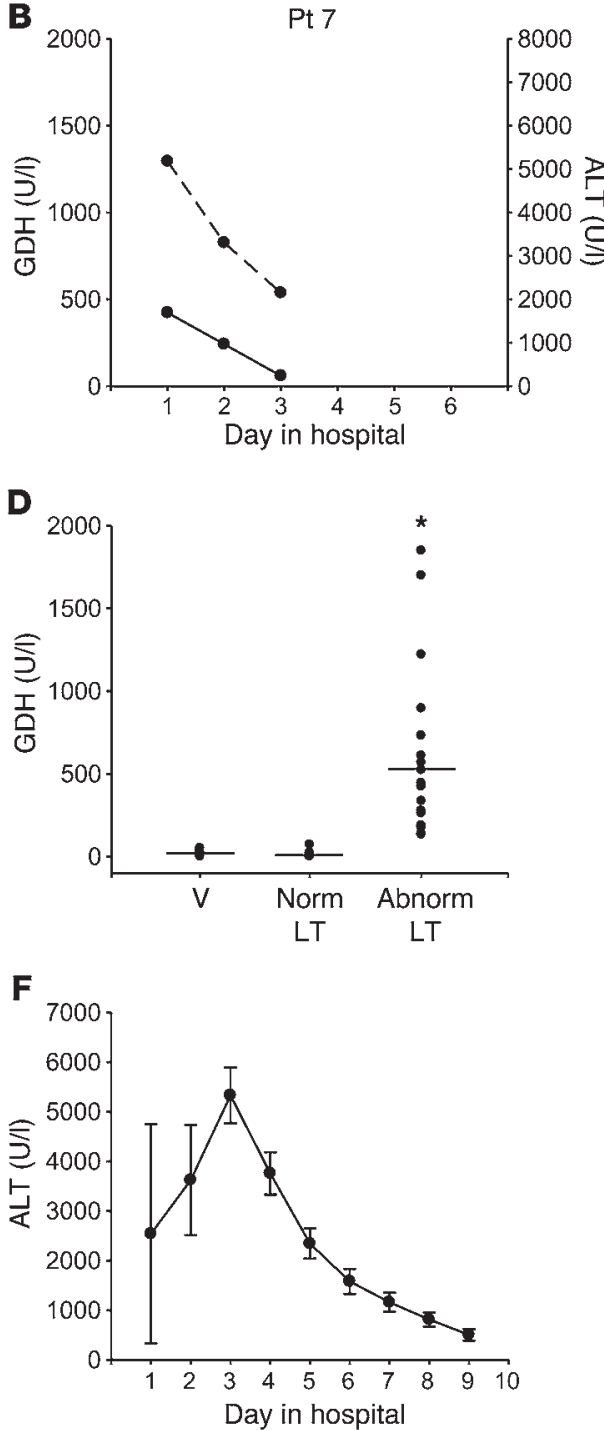

\section{Figure 1}

GDH activity in plasma from APAPoverdose patients. GDH activity was measured in the plasma from APAP-overdose patients throughout their time in the hospital. (A-C) Time course data from 3 representative patients showing both GDH and ALT activity. (D) Plasma GDH activity at the time of peak ALT in healthy volunteers $(\mathrm{V})$, normal $\mathrm{LT}$ group (Norm LT), and abnormal LT group (Abnorm LT). (E) Average plasma GDH activity over time for the patients with abnormal LT results. (F) Average plasma ALT activity over time for patients with abnormal LT results. Data are expressed as mean \pm SEM for $n=20 .{ }^{*} P<0.05$ compared with healthy volunteers.
Mitochondrial DNA in plasma from overdose patients. Mitochondrial DNA (mtDNA) has been shown to be released into circulation after hemorrhagic shock in rats (35) and other physical trauma in humans (36). In addition to serving as another marker of mitochondrial injury, mtDNA appears to act as a damage-associated molecular pattern (DAMP) capable of activating neutrophils and other cells of the innate immune system via TLRs (35-38). To determine whether or not APAP hepatotoxicity leads to release of mtDNA into circulation in humans, mtDNA concentrations were determined in the plasma of these patients using absolute quantification real-time PCR. Primers were designed for subunits of complex I (NADH dehydrogenase) and complex III (cytochrome $c$ oxidase) of the electron transport chain, which are exclusively encoded in mtDNA (39). The concentration of mtDNA in the plasma of overdose patients with abnormal liver function increased over time (Figure 2, A and B), and peak levels correlated with peak ALT (Pearson's coefficient $=0.54, P<0.02)($ Figure $2 \mathrm{C})$. While mtDNA was nearly undetectable in plasma from healthy volunteers and the normal LT group, the average peak mtDNA concentration was $45 \pm 15 \mathrm{ng} / \mathrm{ml}$ in plasma from patients with injury (Figure 2D).
Again, despite the correlation with ALT, there was no significant correlation between mtDNA and PT values in the group with the abnormal LT (data not shown).

Nuclear DNA fragmentation in plasma from overdose patients. Nuclear DNA fragmentation has been demonstrated in mice following treatment with toxic doses of APAP and is important in the mechanism of hepatotoxicity in those animals $(22,40)$. Moreover, because the endonucleases responsible for nuclear DNA fragmentation come from the mitochondrial intermembrane space during mitochondrial dysfunction (22), nuclear DNA fragmentation can be considered an additional indirect marker of mitochondrial dysfunction. We observed an increase in nuclear DNA fragments in plasma over time in the patients of the abnormal LT group using an antihistone ELISA with an anti-DNA secondary antibody (Figure 3). Again, this closely followed ALT activity (Figure 3, A-C). Together with the GDH and mtDNA data, these results provide evidence for release of mitochondrial contents and nuclear DNA fragments into the cytosol with subsequent release into peripheral blood after cell death.

Lack of caspase activation in APAP-overdose patients. It is generally believed that the primary mode of cell death in APAP hepatotoxic- 
A
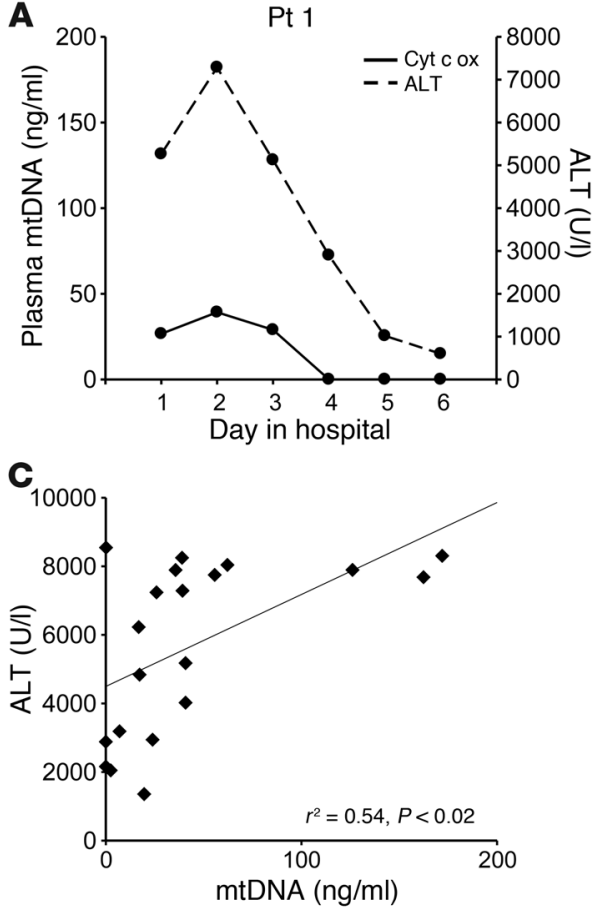

B

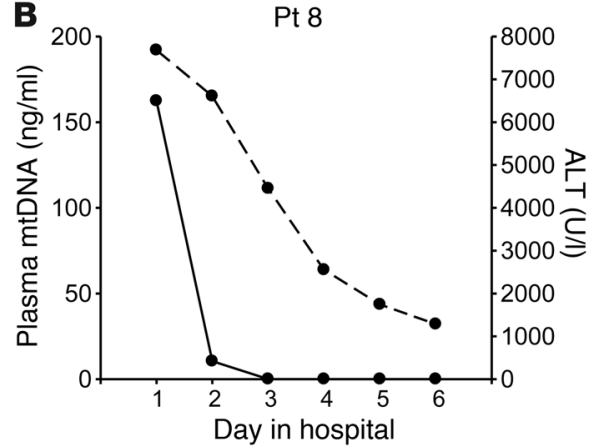

D

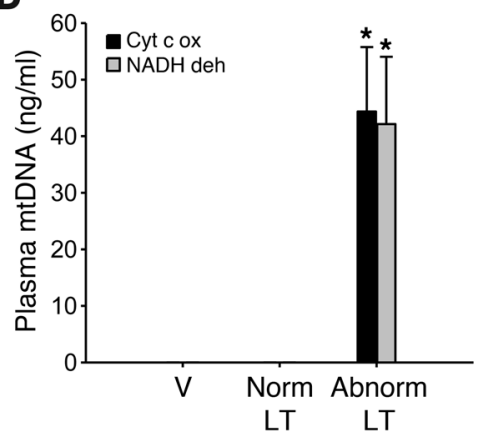

\section{Figure 2}

mtDNA in plasma from APAP-overdose patients. The concentration of mIDNA was determined in the plasma of patients after APAP overdose. Concentrations were measured by absolute quantification real-time PCR using primers for subunits of complex I (NADH deh) and complex IV (Cyt c ox) of the electron transport chain exclusively encoded in mtDNA. (A and $\mathbf{B}$ ) Time course data from 2 representative patients $(\mathrm{Pt})$ showing both mtDNA concentration (Cyt c ox) and ALT activity in plasma. (C) Scatterplot of peak plasma ALT activity against peak mtDNA (Cyt c ox) concentration in plasma from patients of the abnormal LT group. Pearson's correlation coefficient is shown. (D) Average mtDNA concentrations in plasma from healthy volunteers $(n=6)$, normal LT group $(n=20)$, and abnormal LT group $(n=20)$, expressed as mean \pm SEM. ${ }^{*} P<0.05$ compared with healthy volunteers. ity in rodents is oncotic necrosis (41). To determine whether or not apoptosis could be involved in the pathophysiology in humans, caspase- 3 activity and caspase- 3 protein levels were measured in plasma. In the normal LT group, no caspase- 3 activity or caspase- 3 protein was detectable (Figure 4, A and B). In patients with APAPinduced liver injury, pro-caspase- 3 was clearly present in plasma (Figure 4A), but neither the active, low-molecular weight fragments nor an increase in enzyme activity was detectable (Figure 4, $A$ and B). Similar to these findings in patients, no caspase- 3 protein was observed in plasma of control mice. APAP-induced liver injury resulted in the selective appearance of the proenzyme in plasma (Figure 4A), but again no low-molecular weight forms could be seen. In contrast, mice treated with galactosamine and endotoxin $(\mathrm{G} / \mathrm{E})$ as a positive control for apoptosis not only had detectable pro-caspase- 3 in plasma, but also an active fragment (Figure 4A). Consistent with this observation, substantial caspase- 3 enzyme activity was present (Figure 4B). Thus, if there is relevant apoptotic cell death in the liver, as after G/E treatment (42), both caspase- 3 enzyme activity and active caspase- 3 fragments are found in plasma. The absence of these parameters in human and mouse plasma after APAP overdose suggests that apoptosis is not a relevant mechanism of cell death.

Release of mitochondrial markers is not a secondary effect of cell necrosis. It is possible that mitochondria and mitochondrial contents will be released into blood and become detectable in plasma as a result of oncotic necrosis. To verify that high levels of GDH and mtDNA in plasma reflect the mechanism of APAP hepatotoxicity and are not merely a secondary effect of oncotic cell death, these parameters were measured in a model of liver necrosis not thought to involve mitochondria. Furosemide is a loop diuretic prescribed for cases of hypertension and congestive heart failure. At high doses, furosemide can cause liver injury without affecting mitochondrial function (43). Mice treated with this drug had a significant increase in ALT activity in serum at 24 hours (Figure
$5 \mathrm{~A})$, with centrilobular necrosis similar to APAP-induced injury (Figure 6). Although there was a minor increase in GDH activity over controls at this time point, the difference was not statistically significant (Figure 5A). Similarly, serum mtDNA concentrations were only slightly elevated compared with the extensive increase in APAP-treated mice (Figure 5B). These data indicate that high levels of plasma GDH and mtDNA are indicative of the mechanism of APAP toxicity and are not simply a result of tissue necrosis.

Plasma biomarkers and tissue correlation. Because no liver biopsy samples can be obtained from patients during the early phase of liver injury after APAP overdose, we had to rely on plasma biomarkers. To test how well plasma biomarkers reflect events in the tissue, mice were treated with $300 \mathrm{mg} / \mathrm{kg}$ APAP. Blood and livers were collected at several time points, and plasma biomarkers were assayed. Liver histology revealed a close correlation between the development of areas of necrosis in the tissue and the time course of ALT activity in plasma (Figure 7, A and B). Similarly, TUNEL staining (for DNA fragmentation) in tissue sections was followed closely by the rise in nuclear DNA fragments in mouse plasma (Figure 7, C and D). These data show that plasma biomarkers accurately reflect events in the tissue injury after APAP overdose in mice. It seems reasonable to assume that the same relationship between plasma biomarkers and tissue exists in patients.

\section{Discussion}

The objective of this investigation was to gain further insight into the intracellular mechanisms of APAP hepatotoxicity in humans and to assess the mode of cell death. Because there is no diagnostic benefit that would justify the risk of a liver biopsy during the early injury phase after APAP overdose, our approach was to use plasma biomarkers, validated in a mouse model, to obtain reliable mechanistic information. We studied 3 groups of patients. In addition to an age- and sex-matched control group, APAP-overdose patients were divided into 2 groups: one with evidence of liver injury (peak 

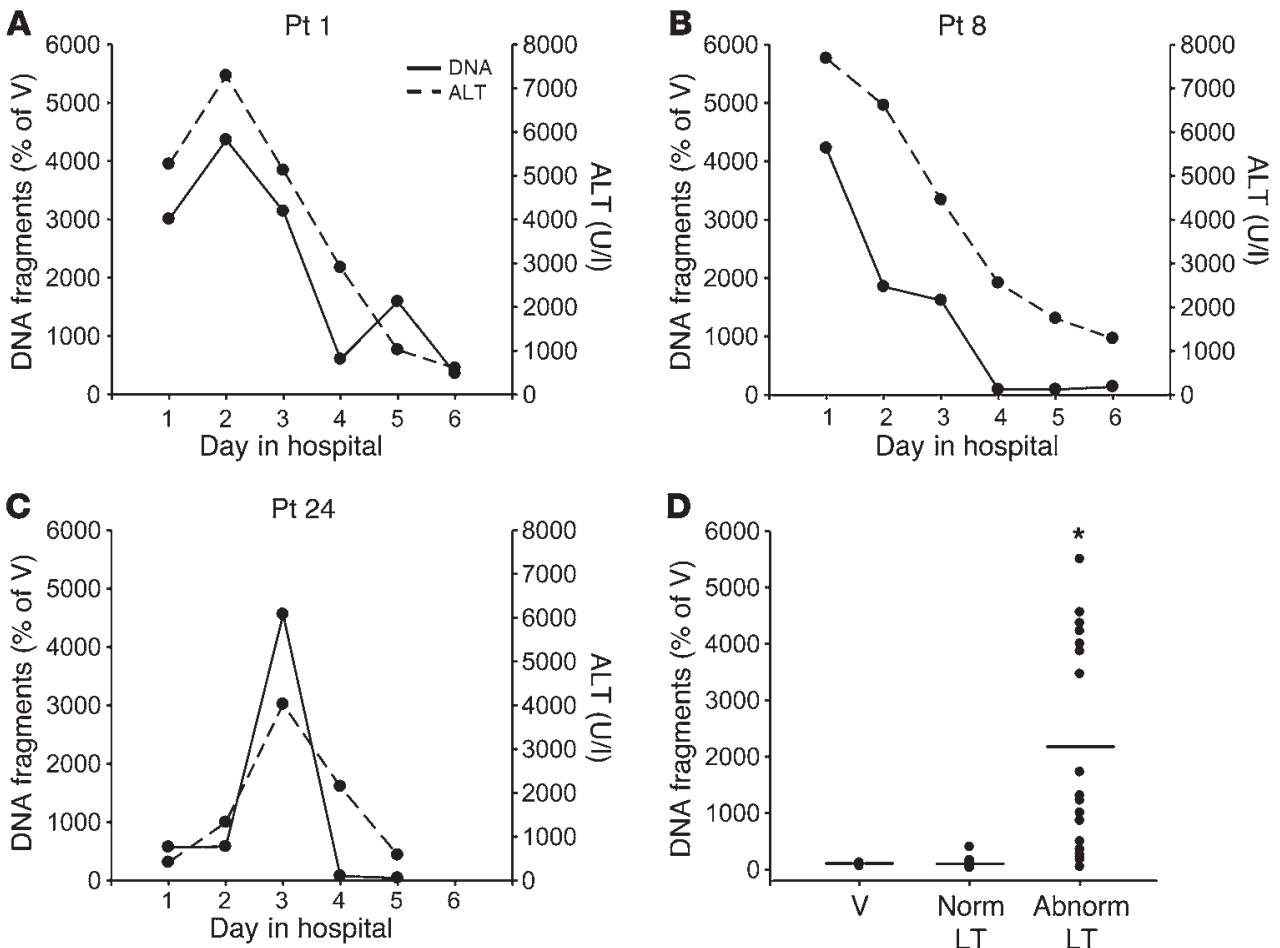

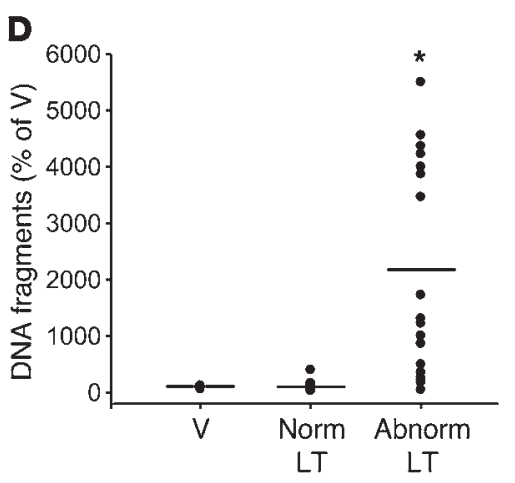

\section{Figure 3}

Nuclear DNA fragments in plasma from APAP-overdose patients. Nuclear DNA fragments were measured by ELISA in plasma from patients after APAP overdose as an indirect marker of mitochondrial injury. (A-C) Time course data from 3 representative patients showing both DNA fragments (expressed as percentage of the average change in OD/min with plasma from healthy volunteers) and ALT. (D) Average DNA fragments, expressed as percentage of the average change in $\mathrm{OD} / \mathrm{min}$ with plasma from healthy volunteers, for healthy volunteers $(n=6)$, for the normal LT group $(n=20)$, and the abnormal LT group $(n=20)$. Data are expressed as mean \pm SEM. ${ }^{*} P<0.05 \mathrm{com}$ pared with healthy volunteers. plasma ALT activities $\geq 1,000 \mathrm{U} / 1$ and peak $\mathrm{PT} \geq 18 \mathrm{~s}$ ) and one with no or very limited liver injury (PT < $18 \mathrm{~s}$ and ALT $<1,000 \mathrm{U} / 1$; highest: $17.5 \mathrm{~s}$ and $158 \mathrm{U} / \mathrm{l}$, respectively). The low injury in this group was likely due, in part, to early admission and treatment prior to development of toxicity. This would be consistent with the higher plasma APAP levels on admission in the group with normal LTs. Additionally, some of these patients may have taken lower overdoses.

Mitochondrial dysfunction and APAP hepatotoxicity. Mitochondrial dysfunction after toxic doses of APAP has been recognized in rodents since the 1980s, when inhibition of mitochondrial respiration and depletion of ATP were first described $(15,16,44)$. More recent studies have shown the development of oxidative and nitrosative stress within mitochondria and occurrence of the mitochondrial MPT after APAP treatment (18-21). Using a human cell line with high expression levels of CYP (HepaRG), mitochondrial dysfunction has also been shown to occur in cultured human hepatocytes (25). We reasoned that, if mitochondrial membrane integrity is compromised and mitochondrial contents leak into the cytosol in patients, these mitochondrial components must be released into the circulation when the hepatocytes become necrotic. Our data showing the presence of the specific mitochondrial components GDH and mtDNA in plasma of patients with highly elevated ALT levels provide evidence that mitochondrial dysfunction occurs in humans after APAP overdose. The high activity of GDH in the plasma from these patients is consistent with the higher GDH expression in zone 3 of the liver (34), where APAP causes the greatest tissue injury.

\section{Figure 4}

Caspase-3 activation is not detectable in plasma from APAPoverdose patients. (A) Caspase-3 processing was assessed by immunoblot in plasma from patients or from mice treated with either APAP or G/E or from control mice. The human and mouse samples were run on different gels but probed with the same antibody. Full-length $(32 \mathrm{kDa})$ pro-caspase- 3 was detectable in samples from the abnormal LT patient group, as well as from mice treated with APAP or G/E. Cleaved active caspase-3 fragment (17 kDa) was detectable only in plasma from the positive control G/E-treated mice. (B) Average caspase- 3 activity in plasma from $\mathrm{G} / \mathrm{E}$-treated mice $(n=3)$, healthy volunteers $(n=6)$, normal LT group $(n=20)$, and abnormal LT group $(n=20)$ based on cleavage of the caspase-3 substrate Ac-DEVD-AMC. Expressed as mean \pm SEM. ${ }^{\star} P<0.05 \mathrm{com}$ pared with healthy volunteers.

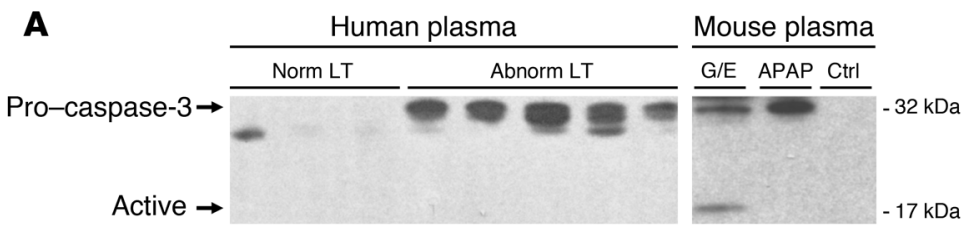

B

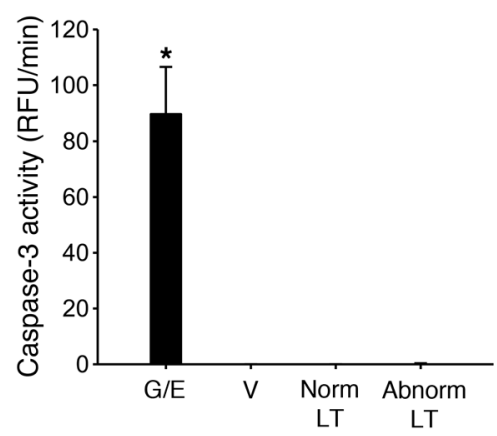



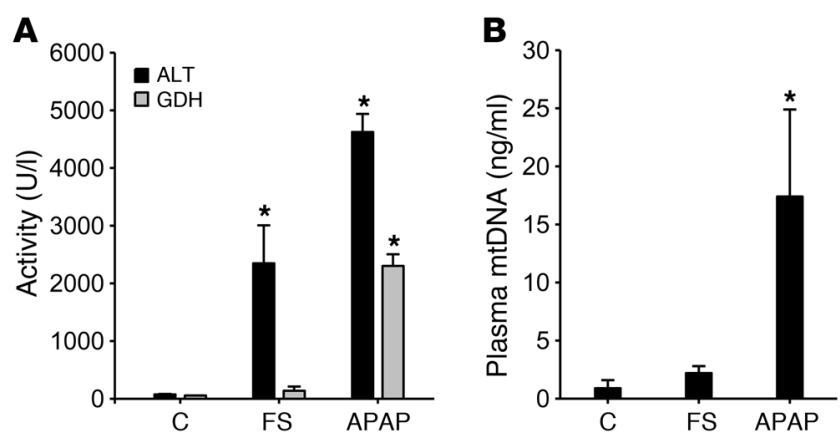

The assumption behind these mechanistic conclusions is that GDH and mtDNA release occurs only when mitochondrial damage is involved, not just cell injury. This hypothesis was confirmed by demonstrating that liver cell damage caused by furosemide, a hepatotoxicant not believed to affect mitochondria (43), resulted in ALT release, but not a statistically significant increase in either mtDNA or GDH in mouse plasma. In contrast, APAP overdose triggers release of both ALT and large amounts of GDH and mtDNA into the plasma of mice. Thus, it is justified to conclude that APAP-induced liver injury in humans involves mitochondrial damage. The time course of the release of GDH and mtDNA correlated well with the appearance of ALT in plasma of all patients, suggesting that mitochondrial damage may be closely related to cell necrosis. In experimental animals, interventions that restored the scavenging capacity for ROS and peroxynitrite in mitochondria $(30,45-47)$ or prevented the MPT (19-21) protected against APAP-induced liver injury. In addition, selective impairment of mitochondrial antioxidant defenses (partial MnSOD deficiency) aggravated APAP hepatotoxicity (48). Moreover, mitochondrial dysfunction and oxidant stress preceded cell necrosis by several hours in murine hepatocytes and in human HepaRG cells (25, 49). Thus, mitochondrial damage is central to APAP-induced cell death in murine models and in a human hepatocyte cell line (19, $21,25,49,50)$. Based on the close correlation between release of biomarkers of mitochondrial damage and cell necrosis, it is likely that mitochondrial dysfunction is a main determinant of liver cell damage in APAP-overdose patients. However, despite these close correlations, our data do not establish that mitochondrial damage is the cause of cell death in humans.

Nuclear DNA damage and APAP hepatotoxicity. In addition to the biomarkers of mitochondrial damage, nuclear DNA fragments were detectable in plasma of patients with severe APAP-induced liver injury. The assay for DNA fragments is based on detection of nuclear histones, which are not present in mtDNA (39). Thus, the antihistone ELISA measures specifically nuclear DNA fragments. No plasma DNA fragments were detectable in healthy volunteers or in patients without severe liver injury. In contrast, the time course of plasma DNA fragment levels in patients with liver injury

\section{Figure 6}

APAP and furosemide both cause centrilobular necrosis. Mice were treated with APAP or furosemide to cause liver necrosis, and liver sections were stained with $\mathrm{H} \& \mathrm{E}$ near the peak of injury for each model (24 hours for FS and 12 hours for APAP). (A and B) Representative images of livers from untreated control mice or ( $\mathbf{C}$ and $\mathbf{D}) 12$ hours after $300 \mathrm{mg} / \mathrm{kg}$ APAP or (E and F) 24 hours after $400 \mathrm{mg} / \mathrm{kg}$ FS. Original magnification, $\times 50(\mathbf{A}, \mathbf{C}$, and E); $\times 100(\mathbf{B}, \mathbf{D}$, and F).

\section{Figure 5}

Release of GDH and mtDNA into blood is not a secondary effect of necrosis. Mice were treated with APAP or furosemide (FS) to cause liver necrosis, and mitochondrial markers were measured in plasma from these animals near the peak of injury for each model (24 hours for FS and 12 hours for APAP). (A) Average GDH and ALT activity. (B) Average mtDNA concentration (Cyt c ox). Data are expressed as mean \pm SEM for $n=3-6$. ${ }^{\star} P<0.05$ versus control.

closely followed the release of ALT, i.e., cell necrosis. A comparison of plasma DNA fragments and nuclear DNA damage in mouse liver after APAP overdose revealed that both parameters correlate with ALT release, suggesting that nuclear DNA damage occurs along with cell death.

Previous studies in rodents documented that DNA fragments after APAP are indistinguishable from apoptotic DNA fragments $(40,51)$. In addition, no nitrotyrosine residues were detectable in the nucleus (51). This indicated that DNA damage was not caused by oxidant stress or peroxynitrite formation, but involved endonucleases. Because of the lack of relevant caspase activation during APAP hepatotoxicity $(23,52)$, the traditional caspase-activated DNase can be excluded. Instead, EndoG and AIF are released from the mitochondrial intermembrane space and translocate to the nucleus during APAP-induced cell death (22). Protection against mitochondrial oxidant stress or prevention of the MPT eliminated nuclear DNA fragmentation $(21,49,51)$. In a later study, the Bcl-2 family member Bax, which forms pores in the outer mitochondrial membrane during the early phase after APAP exposure, was found to facilitate release of EndoG and AIF from mitochondria
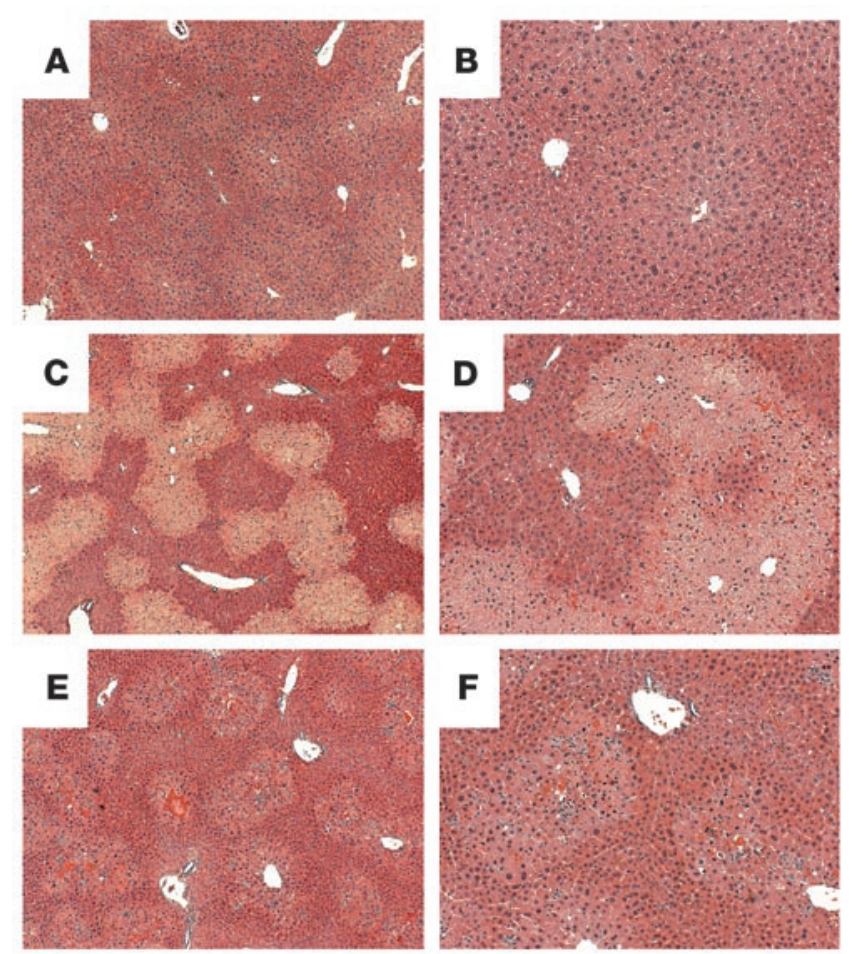
A

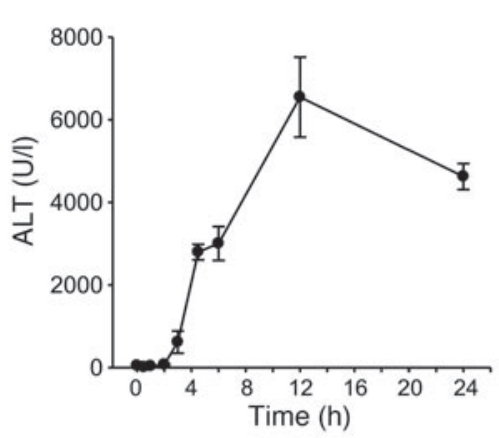

C

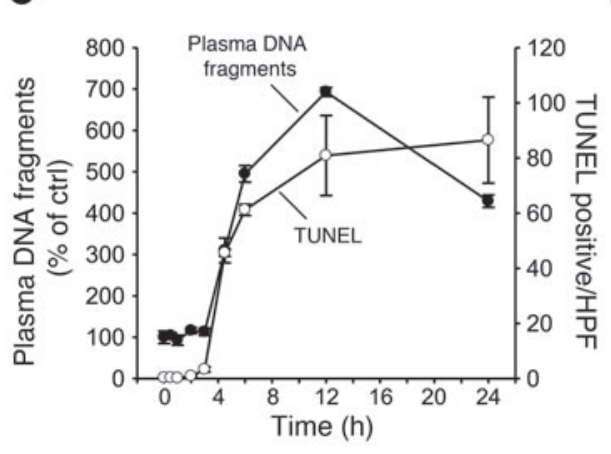

B

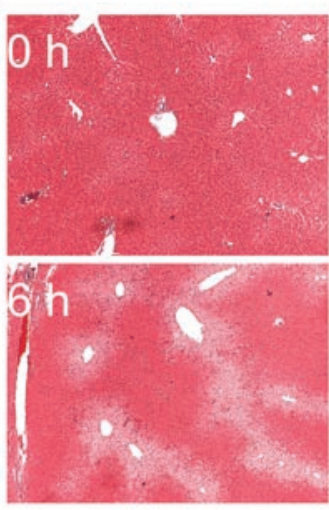

D

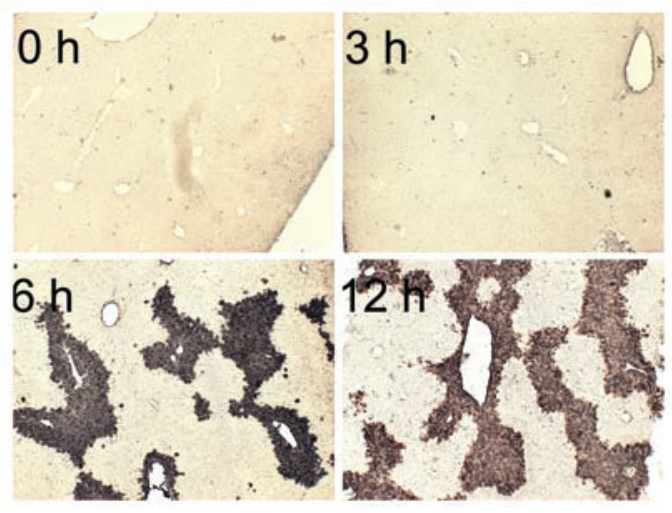

Figure 7

ALT activity and DNA fragments in plasma correlate with tissue injury. Mice were treated with $300 \mathrm{mg} / \mathrm{kg}$ APAP. At various time points, plasma was collected for measurement of ALT and DNA fragments and tissues were collected for histology. (A) Time course of ALT activity in plasma from APAP-treated mice. (B) H\&E sections of liver from APAP-treated mice. Necrosis is evident as pale eosinophilic staining and loss of hepatocyte nuclei. (C) Time course of DNA fragments in plasma from APAPtreated mice (closed circles) and of TUNEL staining in tissue (expressed as TUNEL-positive cells per high power field [HPF]). (D) Representative sections from TUNEL-stained livers over time. Original magnification, $\times 50$. Data are expressed as mean \pm SEM; $n=6$ per time point. and nuclear translocation, which triggered the initial DNA damage (53). Partial AIF deficiency reduced the mitochondrial oxidant stress, nuclear translocation of AIF, and DNA fragmentation (54). There is also evidence that a general endonuclease inhibitor attenuated APAP-induced cell death (55). Together, these findings indicate that nuclear DNA damage is dependent on mitochondrial dysfunction and the release of endonucleases from the intermembrane space. Thus, nuclear DNA damage is closely related and even contributes to liver cell death in the murine model. Given the similar appearance of nuclear DNA in patients with APAP-induced liver injury, it is likely that the same mechanisms of DNA damage apply in human liver.

DNA as DAMP. Our data indicate that nuclear DNA fragments and $\mathrm{mtDNA}$ are released into the plasma of patients and in mice after APAP overdose. These molecules can act as DAMPs through activation of TLRs, especially TLR9, to induce cytokine formation after APAP (56). In the mouse model, there is extensive formation of proinflammatory mediators and recruitment of neutrophils into the liver in response to APAP overdose (57). However, the preponderance of the experimental evidence argues against a relevant contribution of neutrophils to the injury process (58). Consistent with these findings, neutrophils isolated from the injured liver or from the blood are not activated during the main injury phase of APAP hepatotoxicity in mice (59). Preliminary data for neutrophil activation in APAP-overdose patients appear to confirm the lack of neutrophil activation during the injury phase, but show a progressive activation at later time points (C.D. Williams and H. Jaeschke, unpublished observations). Thus, it is likely that in patients, the release of DAMPs, such as nuclear DNA fragments and mtDNA during the injury phase, contributes to activation of innate immune cells, which are involved in the removal of necrotic cell debris and thus contribute to the recovery as observed in mice (58).

Mode of APAP-induced cell death in patients. It is generally accepted that the mode of APAP-induced liver cell death in mice is oncotic necrosis. This is based on morphological evidence (cell swelling, vacuolization, karyorrhexis, and karyolysis), the massive release of cell contents (ALT), and the resulting inflammation (23). However, there is limited evidence of apoptosis. Hallmarks of apoptotic cell death include several morphological features, such as cell shrinkage, chromatin condensation, and formation of apoptotic bodies as well as extensive caspase activation (60). In general, only very few apoptotic cells are detectable after APAP overdose (23), and there is no relevant caspase activation in mouse livers $(23,52,61)$. Furthermore, pancaspase inhibitor did not protect against APAP hepatotoxicity $(23-25,52,62,63)$. However, most of these experiments were done with overnight-fasted animals. Recently, it was suggested that in fed mice with higher cellular ATP content, APAP causes limited caspase activation and some apoptotic cell death (62). This conclusion was also based on the detection of a K18 cleavage product, which is thought to be specific for caspase- 3 activity (62). The use of this assay has resulted in conflicting data in the clinical literature. A case report showed no significant increase of the cleavage product over the time course of injury and recovery of an APAP-overdose patient (64). In contrast, 2 studies found significant increases of this caspase cleavage product in APAP-overdose patients $(32,33)$. However, as noted by Volkmann et al. (33), necrotic full-length K18 was the dominant form, suggesting that apoptosis plays a relatively minor part in the mechanism of cell death after APAP overdose in humans. Our data directly analyzing caspase- 3 activity or the active fragment of caspase- 3 by Western 
blotting did not reveal any evidence for caspase- 3 activation in these patients. However, in the overdose patients with extensive liver injury, pro-caspase-3 protein was present in plasma, reflecting the release of cell contents of necrotic cells. These clinical data are similar to our observations in APAP-treated mice, which had an increase in plasma pro-caspase-3 levels, but no active fragments and no increase in enzyme activity. In contrast, in the G/E model, which is a positive control for caspase-dependent apoptosis in hepatocytes (42), extensive caspase- 3 activity and the active fragment of caspase- 3 were clearly detectable. In a similar experiment, caspase-3 activity was readily detectable in plasma after galactosamine-induced apoptosis in rat liver despite the fact that only $5 \%-6 \%$ of hepatocytes were apoptotic (65). Based on these experiments, it can be concluded that the absence of active caspase- 3 fragments and of detectable caspase- 3 activity in plasma of APAPoverdose patients with severe liver injury suggests that apoptotic cell death is not relevant for the overall pathophysiology. The discrepancy between the detection of minor levels of caspase-dependent K18 cleavage product in some studies $(32,33)$ and our lack of direct plasma caspase- 3 activity measurement in APAP patients requires further study. This could be related to differences in assay sensitivity or potentially even specificity, i.e., K18 could be cleaved by other proteases. However, there is principal agreement among all studies that necrotic cell death is dominant in these patients.

Currently, much emphasis is placed upon the development of novel biomarkers to aid in both the diagnosis and prognosis of various liver diseases. It is possible that mitochondrial markers such as $\mathrm{GDH}$ and mtDNA can also predict outcome. In this study, only one patient did not survive, and none received a liver transplant. With these numbers, we are unable to assess the prognostic value of these biomarkers. Larger cohorts will be needed to determine whether or not these biomarkers are predictive of patient outcome.

In summary, our data demonstrated the release of biomarkers reflecting mitochondrial damage and nuclear DNA fragmentation in patients with severe APAP-induced liver injury. These events led to predominantly necrotic cell death. The use of these biomarkers and the mechanistic conclusions were extensively validated by parallel studies in mice in which tissue injury and the release of these markers were measured. Our findings provide strong support for the hypothesis that mitochondrial dysfunction and DNA damage are critical events in the mechanism of cell necrosis after APAP overdose in patients. In addition, these data confirm that the in vivo mouse model, primary murine hepatocytes, and human HepaRG cells are appropriate experimental systems to study cell death mechanisms that appear to be relevant for APAP-overdose patients.

\section{Methods}

Patient selection. Patients admitted to the University of Kansas Hospital or to the Banner Good Samaritan Medical Center following APAP overdose were studied prospectively. The study design and protocol were approved by the respective institutional review boards (IRB), with regular follow-up assessment. Patients presented to the emergency department or were admitted to the intensive care unit with evidence of APAP overdose. The diagnosis was made by a physician on site, and all participants were required to sign a consent form. The patient population included both acute- and chronicoverdose patients. Whereas all patients in the normal LT group were acuteoverdose cases, 5 out of 20 patients in the group with abnormal LTs took repeated doses in excess of the maximum recommended therapeutic dose of $4 \mathrm{~g} / \mathrm{d}$ over multiple days. The inclusion criteria were patient-reported history of APAP overdose, high-serum APAP levels, and abnormal LTs (based on ALT, aspartate aminotransferase [AST], PT, bilirubin). All of the participants fulfilled at least 2 of these criteria. Each patient's blood and/or urine was subjected to a toxicology screen: in addition to APAP, various levels of ethanol, cocaine, antidepressants, or other drugs were detected in some patients. However, there was no medication found besides APAP that could have acutely caused severe liver injury. Patients were excluded if there was reasonable evidence for liver injury due to another cause (i.e., viral hepatitis, ischemic liver, etc.) All overdose patients received standard of care NAC treatment. For each individual, age and sex were recorded. APAP, ALT, AST, PT, creatinine, and total bilirubin levels in serum were monitored.

Animal studies. Male C57BL/6 mice were purchased from The Jackson Laboratory and housed in an environmentally controlled room with a 12-hour light/12-hour dark cycle and ad libitum access to food and water. For APAP treatment, mice were fasted overnight before receiving an i.p. injection of $300 \mathrm{mg} / \mathrm{kg}$ APAP in warm $0.9 \%$ saline. Other animals fed ad libitum were treated i.p. with $400 \mathrm{mg} / \mathrm{kg}$ furosemide dissolved in warm phosphate-buffered saline, $\mathrm{pH} 7.5-8.0$, without fasting. As positive controls for caspase- 3 activation, some fed mice received $700 \mathrm{mg} / \mathrm{kg}$ galactosamine and $100 \mu \mathrm{g} / \mathrm{kg}$ endotoxin to induce hepatocellular apoptosis. All chemicals were purchased from Sigma-Aldrich. At the indicated times, animals were killed by cervical dislocation. After blood collection, serum was obtained by centrifugation at $14,000-20,000 \mathrm{~g}$ for $15-20$ minutes. Livers were excised, and small sections were fixed in $10 \%$ phosphate-buffered formalin for histological analysis.

Clinical assays. ALT levels in serum were measured in the hospital and later confirmed in our laboratory using an ALT kit (Pointe Scientific). AST, coagulation parameters, and bilirubin were also measured on site using standard protocols.

GDH activity. GDH activity was determined using a modified version of the method of Passonneau and Lowry (66). Briefly, aliquots of 10-100 $\mu \mathrm{l}$ plasma were mixed in $700 \mu \mathrm{l}$ of $200 \mathrm{mM}$ imidazole buffer with $25 \mathrm{mM}$ ammonium acetate, $200 \mu \mathrm{M} \mathrm{NADH}, 100 \mu \mathrm{M}$ ADP, and $0.05 \%$ bovine serum albumin, $\mathrm{pH}$ 8.0. The disappearance of NADH was monitored at $340 \mathrm{~nm}$ to obtain a baseline reading, and then $50 \mu \mathrm{l}$ of a $2 \mathrm{mM} \alpha$-ketoglutarate solution was added to begin the GDH reaction. The baseline activity was then subtracted from the GDH activity.

DNA fragmentation. DNA fragmentation was measured using an antihistone ELISA with an anti-DNA secondary antibody, according to the manufacturer's instructions (Roche). In addition, liver sections were stained with H\&E for evaluation of necrosis and with TUNEL in situ cell death assay (Roche) for visualization of DNA strand breaks as previously described (23).

Caspase activation. Pro-caspase-3 processing was assessed by Western blotting with a single antibody, cross-reactive with both human and mouse caspase-3 (Cell Signaling). Caspase activity was measured as Z-VAD-FMK-inhibitable cleavage of the caspase-3 substrate Ac-DEVD-AMC, as described (42).

MtDNA. mtDNA was measured by absolute quantification real-time PCR, as described (36). Total DNA was isolated from plasma samples using a QIAamp Blood and Mini Kit (QIAGEN), which removes plasma components with the potential to interfere in PCR analysis. The samples were then diluted, and the same amount of total DNA was added to each reaction on each plate. $\mathrm{mtDNA}$ was determined using 2 pairs of human primers, 1 for human NADH dehydrogenase (forward 5'-ATACCCATGGCCAACCTCCT-3' and reverse $5^{\prime}$-GGGCCTTTGCGTAGTTGTAT-3') and 1 for human cytochrome $c$ oxidase subunit III (forward 5'-ATGACCCACCAATCACATGC-3' and reverse $5^{\prime}$-ATCACATGGCTAGGCCGGAG-3'). For mice, another 2 primer pairs were designed: mouse NADH dehydrogenase subunit 6 (forward 5'-CACACAAACATAACCACTTTAAC-3' and reverse $5^{\prime}$-GTAGGTCAATGAATGAGTGGTT-3') and mouse cytochrome $c$ oxidase subunit III (5'-ACCAAGGCCACCACACTCCT-3' and 5'ACGCTCAGAAGAATCCTGCAAAGAA-3'). To construct standard curves, mitochondrial pellets were 
isolated from either human hepatoma cells (HepaRG) or mouse liver by differential centrifugation. Purity of mtDNA standards was verified by realtime PCR using primers for both mitochondrial genes and nuclear-encoded $\beta$-actin. Dilutions of these purified mtDNA samples were prepared and standards were included on each PCR plate for each gene tested. The limit of detection for the assay was determined to be less than $0.05 \mathrm{ng} / \mathrm{ml}$.

Statistics. The Shapiro-Wilk test was used to assess normality. To test for statistical significance, Kruskal-Wallis nonparametric analysis of variance was used for the nonnormally distributed patient data, followed by Dunn's multiple comparisons to test significance between groups. Unpaired 2-tailed Student's $t$ test was used to assess significance between values from control and experimental animals within treated groups. To test for correlation between 2 parameters in patient plasma, Pearson's correlation coefficient was calculated. For all tests, $P<0.05$ was considered significant.

Study approval. The study design was approved by the IRBs of the University of Kansas Medical Center and the Banner Good Samaritan Medical Center. Signed consent was required for all study participants. All experimental protocols for the mouse experiments were approved by the Institutional Animal Care and Use Committee of the University of Kansas Medical Center.

\section{Acknowledgments}

This investigation was supported in part by NIH grants R01 DK070195 and R01 AA12916, a Pilot grant from the Liver Center, University of Kansas Medical Center, and by grants P20 RR016475 and P20 RR021940 from the National Center for Research Resources, and the National Institute of General Medical Sciences (8 P20 GM103549-07) from the NIH. M.R. McGill and C.D. Williams were supported by the Training Program in Environmental Toxicology (T32 ES007079-26A2) from the National Institute of Environmental Health Sciences. In addition, S.C. Curry and H. Jaeschke were supported by grants from McNeil Consumer Health.

Received for publication June 30, 2011, and accepted in revised form January 18, 2012.

Address correspondence to: Hartmut Jaeschke, Department of Pharmacology, Toxicology and Therapeutics, University of Kansas Medical Center, 3901 Rainbow Blvd., MS 1018, Kansas City, Kansas 66160, USA. Phone: 913.588.7969; Fax: 913.588.7501; E-mail: hjaeschke@kumc.edu.
1. Kaufman DW, Kelly JP, Rosenberg L, Anderson TE, Mitchell AA. Recent patterns of medication use in the ambulatory adult population of the United States: the Slone survey. JAMA. 2002;287(3):337-344.

2. Blazer DG, Wu LT. Nonprescription use of pain relievers by middle-aged and elderly communityliving adults: National Survey on Drug Use and Health. J Am Geriatr Soc. 2009;57(7):1252-1257.

3. Wilcox CM, Cryer B, Triadafilopoulos G. Patterns of use and public perception of over-the-counter pain relievers: focus on nonsteroidal anti-inflammatory drugs. J Rheumatol. 2005;32(11):2218-2224.

4. Davidson DG, Eastham WN. Acute liver necrosis following overdose of paracetamol. $\mathrm{Br} \mathrm{Med} \mathrm{J}$. 1966;2(5512):497-499.

5. Larson AM, et al. Acute Liver Failure Study Group. Acetaminophen-induced acute liver failure: results of a United States multicenter, prospective study. Hepatology. 2005;42(6):1364-1372.

6. Bernal W. Changing patterns of causation and the use of transplantation in the United Kingdom. Semin Liver Dis. 2003;23(3):227-237.

7. Gow PJ, Jones RM, Dobson JL, Angus PW. Etiology and outcome of fulminant hepatic failure managed at an Australian liver transplant unit.J Gastroenterol Hepatol. 2004;19(2):154-159.

8. Wei G, et al. Acute liver failure in Sweden: etiology and outcome. J Intern Med. 2007;262(3):393-401.

9. Nourjah P, Ahmad SR, Karwoski C, Willy M. Estimates of acetaminophen (Paracetamol)-associated overdoses in the United States. Pharmacoepidemiol Drug Saf. 2006;15(6):398-405.

10. Nelson SD. Molecular mechanisms of the hepatotoxicity caused by acetaminophen. Semin Liver Dis. 1990;10(4):267-278.

11. Mitchell JR, Jollow DJ, Potter WZ, Davis DC, Gillette JR, Brodie BB. Acetaminophen-induced hepatic necrosis. I. Role of drug metabolism. J Pharmacol Exp Ther. 1973;187(1):185-194.

12. Jollow DJ, Mitchell JR, Potter WZ, Davis DC, Gillette JR, Brodie BB. Acetaminophen-induced hepatic necrosis. II. Role of covalent binding in vivo. J Pharmacol Exp Ther. 1973;187(1):195-202.

13. Potter WZ, Davis DC, Mitchell JR, Jollow DJ, Gillette JR, Brodie BB. Acetaminophen-induced hepatic necrosis. 3. Cytochrome P-450-mediated covalent binding in vitro. J Pharmacol Exp Ther. 1973; 187(1):203-210.

14. Mitchell JR, Jollow DJ, Potter WZ, Gillette JR, Brodie BB. Acetaminophen-induced hepatic necrosis. IV. Protective role of glutathione. J Pharmacol Exp
Ther. 1973;187(1):211-217.

15. Placke ME, Ginsberg GL, Wyand DS, Cohen SD. Ultrastructural changes during acute acetaminophen-induced hepatotoxicity in the mouse: a time and dose study. Toxicol Pathol. 1987;15(4):431-438.

16. Myers LL, Beierschmitt WP, Khairallah EA, Cohen SD. Acetaminophen-induced inhibition of hepatic mitochondrial respiration in mice. Toxicol Appl Pharmacol. 1988;93(3):378-387.

17. Tirmenstein MA, Nelson SD. Subcellular binding and effects on calcium homeostasis produced by acetaminophen and a nonhepatotoxic regioisomer, $3^{\prime}$-hydroxyacetanilide, in mouse liver. J Biol Chem. 1989;264(17):9814-9819.

18. Jaeschke H. Glutathione disulfide formation and oxidant stress during acetaminophen-induced hepatotoxicity in mice in vivo: the protective effect of allopurinol. J Pharmacol Exp Ther. 1990;255(3):935-941.

19. Kon K, Kim JS, Jaeschke H, Lemasters JJ. Mitochondrial permeability transition in acetaminopheninduced necrosis and apoptosis of cultured mouse hepatocytes. Hepatology. 2004;40(5):1170-1179.

20. Masubuchi Y, Suda C, Horie T. Involvement of mitochondrial permeability transition in acetaminophen-induced liver injury in mice.J Hepatol. 2005; 42(1):110-116

21. Ramachandran A, Lebofsky M, Baines CP, Lemasters JJ, Jaeschke H. Cyclophilin D deficiency protects against acetaminophen-induced oxidant stress and liver injury. Free Radic Res. 2011;45(2):156-164.

22. Bajt ML, Cover C, Lemasters JJ, Jaeschke H. Nuclear translocation of endonuclease $\mathrm{G}$ and apoptosisinducing factor during acetaminophen-induced liver injury. Toxicol Sci. 2006;94(1):217-25.

23. Gujral JS, Knight TR, Farhood A, Bajt ML, Jaeschke $\mathrm{H}$. Mode of cell death after acetaminophen overdose in mice: apoptosis or oncotic necrosis? Toxicol Sci. 2002;67(2):322-328

24. Jaeschke H, Cover C, Bajt ML. Role of caspases in acetaminophen-induced liver injury. Life Sci. 2006;78(15):1670-1676.

25. McGill MR, Yan HM, Ramachandran A, Murray GJ, Rollins DE, Jaeschke H. HepaRG cells: a human model to study mechanisms of acetaminophen hepatotoxicity. Hepatology. 2011;53(3):974-982.

26. Lauterburg BH, Mitchell JR. Therapeutic doses of acetaminophen stimulate the turnover of cysteine and glutathione in man.J Hepatol. 1987;4(2):206-211.

27. Slattery JT, Wilson JM, Kalhorn TF, Nelson SD. Dose-dependent pharmacokinetics of acetaminophen: evidence of glutathione depletion in man.
Clin Pharmacol Ther. 1987;41(4):413-418.

28. Muldrew KL, et al. Determination of acetaminophen-protein adducts in mouse liver and serum and human serum after hepatotoxic doses of acetaminophen using high-performance liquid chromatography with electrochemical detection. Drug Metab Dispos. 2002;30(4):446-451.

29. Davern TJ 2nd, et al. Measurement of serum acetaminophen-protein adducts in patients with acute liver failure. Gastroenterology. 2006;130(3):687-694.

30. Saito C, Zwingmann C, Jaeschke H. Novel mechanisms of protection against acetaminophen hepatotoxicity in mice by glutathione and $\mathrm{N}$-acetylcysteine. Hepatology. 2010;51(1):246-254.

31. Smilkstein MJ, Knapp GL, Kulig KW, Rumack BH. Efficacy of oral $\mathrm{N}$-acetylcysteine in the treatment of acetaminophen overdose: Analysis of the national multicenter study (1976 to 1985). N Engl J Med. 1988;319(24):1557-1562.

32. Rutherford AE, et al. Serum apoptosis markers in acute liver failure: a pilot study. Clin Gastroenterol Hepatol. 2007;5(12):1477-1483.

33. Volkmann X, et al. Caspase activation is associated with spontaneous recovery from acute liver failure. Hepatology. 2008;47(5):1624-1633.

34. Racine-Samson L, et al. The metabolic organization of the adult human liver: a comparative study of normal fibrotic, and cirrhotic liver tissue. Hepatology. 1996;24(1):104-113.

35. Zhang Q, Itagaki K, Hauser CJ. Mitochondrial DNA is released by shock and activates neutrophils via p38 map kinase. Shock. 2010;34(1):55-59.

36. Zhang Q, et al. Circulating mitochondrial DAMPs cause inflammatory responses to injury. Nature. 2010;464(7285):104-107.

37. Hemmi H, et al. A toll-like receptor recognizes bacterial DNA. Nature. 2000;408(6813):740-745.

38. Chuang TH, Lee J, Kline L, Mathison JC, Ulevitch RJ. Toll-like receptor 9 mediates CpG-DNA signaling. J Leukoc Biol. 2002;71(3):538-544.

39. Wallace DC. Diseases of the mitochondrial DNA. Annu Rev Biochem. 1992;61:1175-1212.

40. Ray SD, Sorge CL, Raucy JL, Corcoran GB. Early loss of large genomic DNA in vivo with accumulation of $\mathrm{Ca} 2+$ in the nucleus during acetaminophen-induced liver injury. Toxicol Appl Pharmacol. 1990;106(2):346-351.

41. Jaeschke H, Gujral JS, Bajt ML. Apoptosis and necrosis in liver disease. Liver Int. 2004;24(2):85-89.

42. Jaeschke H, Fisher MA, Lawson JA, Simmons CA, Farhood A, Jones DA. Activation of caspase 3 
(CPP32)-like proteases is essential for TNF-alphainduced hepatic parenchymal cell apoptosis and neutrophil-mediated necrosis in a murine endotoxin shock model. J Immunol. 1998;160(7):3480-3486.

43. Wong SG, Card JW, Racz WJ. The role of mitochondrial injury in bromobenzene and furosemide induced hepatotoxicity. Toxicol Lett. 2000;116(3):171-181.

44. Andersson BS, Rundgren M, Nelson SD, Harder S. $\mathrm{N}$-acetyl-p-benzoquinone imine-induced changes in the energy metabolism in hepatocytes. Chem Biol Interact. 1990;75(2):201-211.

45. Knight TR, Ho YS, Farhood A, Jaeschke H. Peroxynitrite is a critical mediator of acetaminophen hepatotoxicity in murine livers: protection by glutathione. J Pharmacol Exp Ther. 2002;303(2):468-475.

46. James LP, McCullough SS, Lamps LW, Hinson JA. Effect of $\mathrm{N}$-acetylcysteine on acetaminophen toxicity in mice: relationship to reactive nitrogen and cytokine formation. Toxicol Sci. 2003;75(2):458-467.

47. Bajt ML, Knight TR, Farhood A, Jaeschke H. Scavenging peroxynitrite with glutathione promotes regeneration and enhances survival during acetaminophen-induced liver injury in mice. J Pharmacol Exp Ther. 2003;307(1):67-73.

48. Ramachandran A, Lebofsky M, Weinman SA, Jaeschke $H$. The impact of partial manganese superoxide dismutase (SOD2)-deficiency on mitochondrial oxidant stress, DNA fragmentation and liver injury during acetaminophen hepatotoxicity. Toxicol Appl Pharmacol. 2011;251(3):226-233.

49. Bajt ML, Knight TR, Lemasters JJ, Jaeschke H. Acetaminophen-induced oxidant stress and cell injury in cultured mouse hepatocytes: protection by N-acetyl cysteine. Toxicol Sci. 2004;80(2):343-349.
50. Jones DP, Lemasters JJ, Han D, Boelsterli UA, Kaplowitz N. Mechanisms of pathogenesis in drug hepatotoxicity putting the stress on mitochondria. Mol Interv. 2010;10(2):98-111.

51. Cover $\mathrm{C}$, et al. Peroxynitrite-induced mitochondrial and endonuclease-mediated nuclear DNA damage in acetaminophen hepatotoxicity. J Pharmacol Exp Ther. 2005;315(2):879-887.

52. Lawson JA, Fisher MA, Simmons CA, Farhood A Jaeschke H. Inhibition of Fas receptor (CD95)induced hepatic caspase activation and apoptosis by acetaminophen in mice. Toxicol Appl Pharmacol. 1999;156(3):179-186.

53. Bajt ML, Farhood A, Lemasters JJ, Jaeschke H. Mitochondrial bax translocation accelerates DNA fragmentation and cell necrosis in a murine model of acetaminophen hepatotoxicity. J Pharmacol Exp Ther. 2008;324(1):8-14.

54. Bajt ML, et al. Apoptosis-inducing factor modulates mitochondrial oxidant stress in acetaminophen hepatotoxicity. Toxicol Sci. 2011;122(2):598-605.

55. Shen W, Kamendulis LM, Ray SD, Corcoran GB. Acetaminophen-induced cytotoxicity in cultured mouse hepatocytes: effects of $\mathrm{Ca}(2+)$-endonuclease, DNA repair, and glutathione depletion inhibitors on DNA fragmentation and cell death. Toxicol Appl Pharmacol. 1992;112(1):32-40.

56. Imaeda AB, et al. Acetaminophen-induced hepatotoxicity in mice is dependent on Tlr9 and the Nalp3 inflammasome. J Clin Invest. 2009;119(2):305-314.

57. Lawson JA, Farhood A, Hopper RD, Bajt ML, Jaeschke $H$. The hepatic inflammatory response after acetaminophen overdose: role of neutrophils. Toxicol Sci. 2000;54(2):509-516.
58. Jaeschke H, Williams CD, Ramachandran A, Bajt ML. Acetaminophen hepatotoxicity and repair: the role of sterile inflammation and innate immunity. Liver Int. 2012;32(1):8-20.

59. Williams CD, Bajt ML, Farhood A, Jaeschke H. Acetaminophen-induced hepatic neutrophil accumulation and inflammatory liver injury in CD18deficient mice. Liver Int. 2010;30(9):1280-1292.

60. Jaeschke H, Lemasters JJ. Apoptosis versus oncotic necrosis in hepatic ischemia/reperfusion injury. Gastroenterology. 2003;125(4):1246-1257.

61. Adams ML, et al. Enhanced acetaminophen hepatotoxicity in transgenic mice overexpressing BCL-2. Mol Pharmacol. 2001;60(5):907-915.

62. Antoine DJ, et al. High-mobility group box-1 protein and keratin-18, circulating serum proteins informative of acetaminophen-induced necrosis and apoptosis in vivo. Toxicol Sci. 2009;112(2):521-531.

63. Williams CD, Farhood A, Jaeschke H. Role of caspase- 1 and interleukin-1beta in acetaminopheninduced hepatic inflammation and liver injury. Toxicol Appl Pharmacol. 2010;247(3):169-178.

64. Bechmann LP, Marquitan G, Jochum C, Saner F, Gerken G, Canbay A. Apoptosis versus necrosis rate as a predictor in acute liver failure following acetaminophen intoxication compared with acute-onchronic liver failure. Liver Int. 2008;28(5):713-716.

65. Gujral JS, Farhood A, Jaeschke H. Oncotic necrosis and caspase-dependent apoptosis during galactosamine-induced liver injury in rats. Toxicol Appl Pharmacol. 2003;190(1):37-46.

66. Passonneau JV, Lowry OH. Enzymatic Analysis. A Practical Guide. Totowa, New Jersey, USA: Humana Press; 1993. 